\title{
Single-cell analysis reveals the pan-cancer invasiveness-associated transition of adipose-derived stromal cells into COL11A1-expressing cancer-associated fibroblasts
}

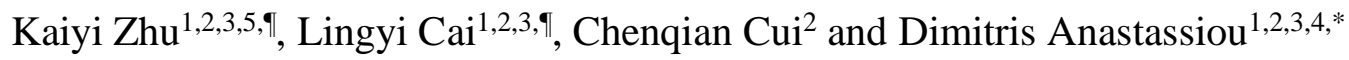

${ }^{1}$ Department of Systems Biology, Columbia University, New York, NY, USA.

${ }^{2}$ Department of Electrical Engineering, Columbia University, New York, NY, USA.

${ }^{3}$ Center for Cancer Systems Therapeutics, Columbia University, New York, NY, USA.

${ }^{4}$ Herbert Irving Comprehensive Cancer Center, Columbia University, New York, NY. USA.

${ }^{5}$ Current affiliation: Icahn School of Medicine at Mount Sinai, New York, NY 10029, USA.

* Corresponding author

E-mail: d.anastassiou@ columbia.edu (DA)

IThese authors contributed equally to this work. 


\begin{abstract}
During the last ten years, many research results have been referring to a particular type of cancerassociated fibroblasts associated with poor prognosis, invasiveness, metastasis and resistance to therapy in multiple cancer types, characterized by a gene expression signature with prominent presence of genes COL11A1, THBS2 and INHBA. Identifying the underlying biological mechanisms responsible for their creation may facilitate the discovery of targets for potential pancancer therapeutics. Using a novel computational approach for single-cell gene expression data analysis identifying the dominant cell populations in a sequence of samples from patients at various stages, we conclude that these fibroblasts are produced by a pan-cancer cellular transition originating from a particular type of adipose-derived stromal cells naturally present in the stromal vascular fraction of normal adipose tissue, having a characteristic gene expression signature. Focusing on a rich pancreatic cancer dataset, we provide a detailed description of the continuous modification of the gene expression profiles of cells as they transition from $A P O D$-expressing adipose-derived stromal cells to COL11A1-expressing cancer-associated fibroblasts, identifying the key genes that participate in this transition. These results also provide an explanation to the well-known fact that the adipose microenvironment contributes to cancer progression.
\end{abstract}

\title{
Author summary
}

Computational analysis of rich gene expression data at the single-cell level from cancer biopsies can lead to biological discoveries about the nature of the disease. Using a computational methodology that identifies the gene expression profile of the dominant cell population for a particular cell type in the microenvironment of tumors, we observed that there is a remarkably continuous modification of this profile among patients, corresponding to a cellular transition. 
Specifically, we found that the starting point of this transition has a unique characteristic signature corresponding to cells that are naturally residing in normal adipose tissue. We also found that the endpoint of the transition has another characteristic signature corresponding to a particular type of cancer-associated fibroblasts with prominent expression of gene COL11A1, which has been found strongly associated with invasiveness, metastasis and resistance to therapy in multiple cancer types. Our results provide an explanation to the well-known fact that the adipose tissue contributes to cancer progression, shedding light on the biological mechanism by which tumor cells interact with the adipose microenvironment. We provide a detailed description of the changing profile during the transition, identifying associated genes as potential targets for pan-cancer therapeutics inhibiting the underlying mechanism.

\section{Introduction}

This research is a follow-up of our previous work in 2010 [1], in which we had identified a stageassociated signature of a particular type of cancer-associated fibroblasts (CAFs) by comparing the gene expression of lower stage with that of higher stage cancer samples. The signature, with prominent presence of genes COL11A1, INHBA and THBS2, was identical across cancer types and would only be present after a particular staging threshold, different in each cancer type, was reached. For example, the signature only appeared in ovarian cancer of at least stage III; in colon cancer of at least stage II; and in breast cancer of at least invasive stage I (but never in carcinoma in situ).

We had observed the striking consistency of the signature across cancer types, which was obvious at that time from bulk microarray data. For example, Table 1 shows the top 15 genes ranked in terms of fold change for three different cancer types (breast [2], ovarian [3], pancreatic [4]) using 
data provided in papers published independently in 2006, 2007, 2008, respectively. The breast cancer data compare invasive ductal carcinoma with ductal carcinoma in situ (supplementary data 3, "up in IDC" of the paper [2]); the ovarian cancer data compare metastatic tissue in the omentum with primary tumor (table 2 of the paper [3]); and the pancreatic data compare whole tumor tissue with normal pancreatic tissue (table 1 of the paper [4]).

Table 1. Top 15 ranked genes in terms of fold change (FC) for three different cancer types revealing the signature of the COL11A1-expressing cancer-associated fibroblasts. Shown are the rankings, reported by the authors, for breast, ovarian and pancreatic cancers. We eliminated multiple entries of the same gene (keeping the one that appears first) and dashes. Genes shared in all three cancer types are highlighted in green, while genes appearing twice are highlighted in yellow.

\begin{tabular}{|l|l|l|l|l|l|l|}
\hline & \multicolumn{2}{l}{ Breast } & \multicolumn{2}{l}{ Ovarian } & \multicolumn{2}{l|}{ Pancreatic } \\
\hline Rank & Gene & FC & Gene & FC & Gene & Log2FC \\
\hline 1 & COL11A1 & 6.5 & COL11A1 & 8.23 & INHBA & 5.15 \\
2 & COL10A1 & 4.07 & COL1A1 & 5.67 & COL10A1 & 5 \\
3 & MFAP5 & 3.73 & TIMP3 & 5.52 & POSTN & 4.92 \\
4 & LRRC15 & 3.61 & FN1 & 5.4 & SULF1 & 4.63 \\
5 & INHBA & 3.44 & INHBA & 4.94 & COL8A1 & 4.6 \\
6 & FBN1 & 3.43 & EFEMP1 & 4.86 & COL11A1 & 4.4 \\
7 & SULF1 & 3.35 & DSPG3 & 4.36 & CTHRC1 & 4.38 \\
8 & GREM1 & 3.35 & COL5A2 & 4.07 & COL1A1 & 4.12 \\
9 & COL5A2 & 3.22 & LOX & 4.03 & THBS2 & 3.97 \\
10 & LOX & 3.22 & MFAP5 & 4.01 & HNT & 3.9 \\
11 & COL5A1 & 3.08 & POSTN & 3.97 & CSPG2 & 3.87 \\
12 & THBS2 & 2.99 & COL5A1 & 3.95 & WISP1 & 3.8 \\
13 & LAMB1 & 2.97 & THBS2 & 3.91 & FN1 & 3.69 \\
14 & FAP & 2.96 & FBN1 & 3.9 & COMP & 3.53 \\
15 & SPOCK & 2.91 & FAP & 3.84 & COL5A2 & 3.38 \\
\hline
\end{tabular}

The four genes COL11A1, INHBA, THBS2, COL5A2 appear among the top 15 in all three sets $\left(P=6 \times 10^{-23}\right.$ by hypergeometric test [5]). The actual $P$ value is much lower than that, because, in 
addition to the above overlap, ten additional genes (COL10A1, COL1A1, COL5A1, FAP, FBN1, FN1, LOX, MFAP5, POSTN, SULF1) appear among the top 15 in at least two of the three sets (and are highly ranked in all three sets anyway). This similarity demonstrates that the signature is welldefined and associated with a universal biological mechanism in cancer. We had also found that gene COL11A1 serves as a proxy of the full signature, in the sense that it is the only gene from which all other genes of the signature are consistently top-ranked in terms of the correlation of their expression with that of COL11A1. Accordingly, we had identified a COL11A1-correlated pan-cancer gene signature, listed in table 4 of [1], which we deposited in GSEA. We had referred to those CAFs as MAFs ("metastasis-associated fibroblasts"), because their presence at the invasive edge of the tumor suggests that metastasis is imminent. To avoid any inaccurate interpretation of the term as implying that such fibroblasts are markers of metastasis that has occurred already, here we refer to them as "COL11A1-expressing CAFs."

Since then, many research results were published connecting one of the genes COL11A1, INHBA, $T H B S 2$ with poor prognosis, invasiveness, metastasis, or resistance to therapy, in various cancer types [6-15]. Furthermore, several designated tumor subtypes were identified in individual cancer types as a result of the presence of those pan-cancer CAFs. For example, the top 15 genes distinguishing the ovarian "mesenchymal subtype" according to [16] are POSTN, COL11A1, THBS2, COL5A2, ASPN, FAP, MMP13, VCAN, LUM, COL10A1, CTSK, COMP, CXCL14, FABP4, INHBA. Similarly, the 24 characterizing genes of the "activated stroma subtype" of pancreatic cancer in fig. 2 of [17] are SPARC, COL1A2, COL3A1, POSTN, COL5A2, COL1A1, THBS2, FN1, COL10A1, COL5A1, SFRP2, CDH11, CTHRC1, PNDC1, SULF1, FAP, LUM, COL11A1, ITGA11, MMP11, INHBA, VCAN, GREM1, COMP. In both cases, the subtype-defining signature is clearly the COL11A1/INHBA/THBS2-expressing CAF signature. 
In this work we show that the origin of those COL11A1-expressing CAFs is a type of adiposederived stromal/stem cells (ASCs) naturally present in the stromal vascular fraction (SVF) of normal adipose tissue, expressing a unique characteristic signature containing fibroblastic markers such as $L U M$ and $D C N$ as well as adipose-related genes, such as $A P O D, C F D$ and $M G P$. This finding explains the stage association of the COL11A1-expressing signature as resulting from the interaction of tumor cells with the adipose microenvironment: Indeed, adipose tissue is encountered when ovarian cancer cells reach the omentum (stage III); after colon cancer has grown outside the colon (stage II); and in breast cancer from the beginning of the spread (stage I, but not in situ stage 0$)$.

Our main computational methodology is attractor analysis (Materials and Methods), and is used in a novel manner for the analysis of rich single-cell RNA sequencing (scRNA-seq) data. The unsupervised attractor algorithm [18] iteratively finds co-expression signatures converging to "attractor metagenes" pointing to the core ("heart") of co-expression. Each attractor metagene is defined by a ranked set of genes along with scores determining their corresponding strengths within the signature, so the top-ranked genes are the most representative of the signature. The attractor algorithm has previously been used successfully for identifying features useful for breast cancer prognosis $[19,20]$. When applied on single cell data from a sample, it identifies the gene expression profiles of the dominant cell populations in the sample. Accordingly, we demonstrate that there is a continuous "ASC to COL11A1-expressing CAF transition."

We used publicly available scRNA-seq data sets from five different cancer types. We first focused on one of those datasets [21] from pancreatic ductal adenocarcinoma, because it is exceptionally rich, containing gene expression profiles from 57,530 cells in 24 tumor samples (T1 T24) and 11 normal control samples (N1-N11). Using attractor analysis, we established that (a) 
the dominant fibroblastic population in each of the 11 normal samples is identical to that of the ASC type described above, and (b) that the gene expression profiles of the dominant fibroblastic population of the 24 tumor samples undergo a gradual change as the transition proceeds, starting from the state of the normal ASCs and passing through a continuum of intermediate states. We also validated these results by applying trajectory inference analysis. Finally, we validated our results in the other cancer types (head and neck, ovarian, lung, breast), suggesting the pan-cancer nature of the ASC to COL11A1-expressing CAF transition.

\section{Results}

\subsection{Pancreatic Ductal Adenocarcinoma (PDAC) dataset}

To find the expression profile of the dominant fibroblastic population in each sample, we applied the attractor algorithm on the set of identified mesenchymal cells (Materials and Methods). We used the general fibroblastic marker gene $L U M$ as seed, and found that all samples yielded strong co-expression signatures involving many genes with big overlap among them. S1 Table shows the top 100 genes for each of the 34 samples (11 normal and 23 tumor samples, excluding sample T20 as it did not contain identified fibroblasts). Genes $L U M, D C N, F B L N 1, M M P 2$, SFRP2 and COL1A2 appear in at least 33 out of the 34 samples, revealing a strong similarity shared by all those fibroblastic expression profiles. This strong overlap is consistent with the continuous transition process, as described below. 
2.1.1 Establishing the presence of COL11A1-expressing CAFs. Because it serves as proxy of the full signature [1], a reliable test for determining if a sample contains the COL11A1-expressing CAFs is to rank all genes in terms of their association with COL11A1 and see if INHBA and THBS2 are top ranked. Indeed, this happens in several tumor samples, as shown in Table 2 for some of them (T23, T11, T6, T15, T18). For each sample, the shown genes are co-expressed in the same cells, because of the high correlations in a single-cell dataset.

Table 2. Ranked COL11A1-associated genes in five PDAC samples.

\begin{tabular}{|c|c|c|c|c|c|c|c|c|c|c|}
\hline Rank & $\mathbf{T} 23$ & MI & T11 & MI & T6 & MI & T15 & MI & T18 & MI \\
\hline 1 & COL11A1 & 1 & COL11A1 & 1 & COL11A1 & 1 & COL11A1 & 1 & COL11A1 & 1 \\
\hline 2 & COL10A1 & 0.3603 & CTHRC1 & 0.2434 & MFAP5 & 0.2353 & MFAP5 & 0.3198 & MFAP5 & 0.3408 \\
\hline 3 & COL12A1 & 0.3383 & MFAP5 & 0.2357 & FNDC1 & 0.1997 & GJB2 & 0.2583 & SUGCT & 0.3379 \\
\hline 4 & COL1A1 & 0.3187 & COL12A1 & 0.2345 & NTM & 0.1912 & COL10A1 & 0.2580 & COL10A1 & 0.2899 \\
\hline 5 & THBS2 & 0.3167 & COL10A1 & 0.2238 & COL8A1 & 0.1877 & INHBA & 0.2561 & C5orf46 & 0.2753 \\
\hline 6 & COL1A2 & 0.3099 & C1QTNF3 & 0.2155 & TWIST1 & 0.1714 & C1QTNF3 & 0.2514 & PPAPDC1A & 0.2668 \\
\hline 7 & COL5A2 & 0.3003 & THBS2 & 0.2123 & COL10A1 & 0.1619 & MATN3 & 0.2505 & NTM & 0.2649 \\
\hline 8 & CTHRC1 & 0.2854 & COL1A2 & 0.2045 & THBS2 & 0.1559 & FNDC1 & 0.2503 & COL8A1 & 0.2534 \\
\hline 9 & FN1 & 0.2781 & COL8A1 & 0.2018 & ITGA11 & 0.1556 & COL8A2 & 0.2411 & INHBA & 0.2430 \\
\hline 10 & COL3A1 & 0.2770 & AEBP1 & 0.2000 & PPAPDC1A & 0.1305 & COL1A1 & 0.2399 & FNDC1 & 0.2264 \\
\hline 11 & INHBA & 0.2746 & LUM & 0.1989 & DIO2 & 0.1298 & COL12A1 & 0.2351 & COL12A1 & 0.2194 \\
\hline 12 & AEBP1 & 0.2688 & COL1A1 & 0.1985 & IGFL2 & 0.1178 & COL8A1 & 0.2325 & IGFL2 & 0.2153 \\
\hline 13 & COL5A1 & 0.2626 & FNDC1 & 0.1963 & SUGCT & 0.1170 & THBS2 & 0.2292 & THBS2 & 0.2094 \\
\hline 14 & VCAN & 0.2457 & SFRP2 & 0.1955 & ADAM12 & 0.1165 & NTM & 0.2257 & CTHRC1 & 0.2026 \\
\hline 15 & MFAP5 & 0.2449 & GJB2 & 0.1879 & C1QTNF3 & 0.1165 & COL1A2 & 0.2220 & SULF1 & 0.2015 \\
\hline 16 & MMP11 & 0.2360 & MATN3 & 0.1817 & ITGBL1 & 0.1109 & GREM1 & 0.2156 & COMP & 0.1926 \\
\hline 17 & COL8A1 & 0.2357 & COL3A1 & 0.1740 & GREM1 & 0.1018 & FN1 & 0.2146 & STMN2 & 0.1926 \\
\hline 18 & COL6A3 & 0.2339 & INHBA & 0.1696 & P4HA3 & 0.1008 & IGFL2 & 0.2141 & WNT2 & 0.1925 \\
\hline 19 & POSTN & 0.2316 & $\mathrm{DCN}$ & 0.1692 & INHBA & 0.1002 & CXCL14 & 0.2112 & MMP11 & 0.1919 \\
\hline 20 & MFAP2 & 0.2275 & CTGF & 0.1691 & COL5A1 & 0.0983 & ITGBL1 & 0.2048 & SPOCK1 & 0.1878 \\
\hline
\end{tabular}


bioRxiv preprint doi: https://doi.org/10.1101/2020.06.23.166066; this version posted February 2, 2021. The copyright holder for this preprint (which was not certified by peer review) is the author/funder, who has granted bioRxiv a license to display the preprint in perpetuity. It is made available under aCC-BY-NC-ND 4.0 International license.

2.1.2 Dominant fibroblastic populations in the tumor samples. Table 3 shows the ranked lists of genes for the attractors in each of the rearranged tumor samples. There is a remarkable continuity in the shown expression profiles. The samples at the right side of the table include COL11A1 at increasingly higher ranks. On the other hand, we identified gene $A P O D$ (Apolipoprotein D) as particularly significant, as it is highly ranked on the left side and its rank gradually decreases as we go to the right. The intermediate tumor samples shown in the middle have cells expressing genes that are top-ranked in both the lists on the left as well as on the right. In other words, these cells are in a genuine intermediate state, rather than being a mixture of distinct subtypes. Furthermore, all genes shown in Table 3 have high strengths and therefore are coexpressed inside the same individual cells in each sample.

Table 3. Rearranged PDAC tumor samples showing the continuously changing pattern of the signature profile.

Genes $A P O D$ and $C O L 11 A 1$ are highlighted in green and red, respectively.

\begin{tabular}{|c|c|c|c|c|c|c|c|c|c|c|c|c|c|c|c|c|c|c|c|c|c|}
\hline \multicolumn{2}{|c|}{ RankT2 } & \multirow{2}{*}{\begin{tabular}{|l} 
T14 \\
DCN
\end{tabular}} & & \multirow{2}{*}{$\begin{array}{l}\text { T3 } \\
\text { MMP2 }\end{array}$} & \multirow{2}{*}{$\begin{array}{l}\text { T10 } \\
\text { PDGFRA }\end{array}$} & \multirow{2}{*}{\begin{tabular}{|l} 
T15 \\
SFRP2
\end{tabular}} & \multirow{2}{*}{$\begin{array}{l}\text { T18 } \\
\text { DCN }\end{array}$} & \multirow{2}{*}{\begin{tabular}{|l} 
T12 \\
MMP2
\end{tabular}} & \multirow{2}{*}{$\begin{array}{l}\text { T6 } \\
\text { COL10A1 }\end{array}$} & \multirow{2}{*}{\begin{tabular}{|l|}
24 \\
DCN
\end{tabular}} & & \multirow{2}{*}{\begin{tabular}{|l} 
T5 \\
PDGFRA
\end{tabular}} & \multirow{2}{*}{\begin{tabular}{|l} 
T4 \\
COL10Al
\end{tabular}} & \multirow{2}{*}{$\begin{array}{l}\text { T7 } \\
1 \text { CYPIB1 }\end{array}$} & \multirow{2}{*}{\begin{tabular}{|l} 
T22 \\
COL1A2
\end{tabular}} & & T11 & T23 & T21 & T17 & T8 \\
\hline 1 & LUM & & & & & & & & & & & & & & & & LUM & COLIA1 & COL10A1 & COL10A1 & \\
\hline 2 & APOD & APOD & APOD & LUM & HTRA3 & LUM & SFRP2 & LUM & PDGFRA & LUM & VCAN & CYP1B1 & SFRP2 & SFRP2 & PDGFRA & DCN & DCN & COL1A2 & CTHRCl & CTHRCl & COL10A1 \\
\hline 3 & VCAN & LUM & LUM & APOD & DPT & DCN & LUM & PDGFRA & SFRP2 & FBLN1 & LUM & SFRP2 & COL1A1 & COL8A1 & THBS2 & RARRES2 & CTHRC1 & COL3A1 & THBS2 & $\mathrm{LL}, \mathrm{I}$ & CREB3L1 \\
\hline 4 & SFRP4 & SFRP4 & IGFI & DCN & APOD & VCAN & C3 & CTHRCI & CYPIB1 & VCAN & PDGFRA & SFRP4 & MMP2 & PDGFRA & MMP2 & CTHRC1 & SFRP2 & COL6A3 & GJB2 & ISLR & RP11-4001 \\
\hline 5 & SFRP2 & TSHZ2 & EFEMP1 & FBLN1 & MEG3 & APOD & MMP2 & ITGBL1 & MMP2 & SFRP4 & COL1A2 & DPT & LUM & COL10A1 & COL1A1 & SFRP2 & COL10A1 I & LUM & SFRP2 & MMP2 & SFRP2 \\
\hline 6 & MMP2 & HTRA3 & DGFRA & VCAN & OMD & FBLN1 & APOD & EFEMPI & VCAN & COL1A2 & EFEMPI & LUM & COL1A2 & SFRP4 & COL3A1 & AEBP1 & RARRES2H & $2 \mathrm{FN} 1$ & COLI1A & COL1A1 & BASP1 \\
\hline 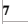 & RARRES1 & FBLN1 & OGN & FBLN5 & ITGBL1 & MMP2 & EFEMP1 & SFRP2 & CTHRC1 & MMP2 & DCN & MEG3 & CTHRC1 & CTHRCl & ITGBL1 & COL10A1 & AEBPI & COL5A2 & CCDC80 & COL1A2 & PDPN \\
\hline 8 & C3 & MMP2 & SFRP4 & PDGFRA & PAPPA & COL6A3 & MFAP4 & FBLN5 & LUM & SFRP2 & CCDC80 & EFEMP1 & DCN & APOD & COL10A1 & NBL1 & NBL1 & VCAN & NBL1 & COL3A1 & $\mathrm{BNC2}$ \\
\hline 9 & MEG3 & COL6A3 & VCAN & SFRP2 & MRC2 & COL1A1 & FBLN1 & VCAN & SFRP4 & $\mathrm{ClR}$ & ISLR & VCAN & CTSK & MMP2 & CTHRCI & MMP2 & CTSK & COL5A1 & DCN & AEBPI & C5orf46 \\
\hline 10 & HTRA3 & VCAN & CTSK & C3 & LSAMP & COL1A2 & SFRP4 & APOD & APOD & CIS & SFRP4 & IGF1 & MFAP2 & PLXDC2 & LUM & CTSK & VCAN & THBS2 & AEBP1 & MMP11 & PLXDC2 \\
\hline 11 & FBLN1 & GPC3 & COL1A2 & MGP & CYPIB1 I & ISLR & CCDC 80 & COL8AI & PLXDC2 & APOD & COL6A3 & FBLN5 & APOD & VCAN & SFRP2 & THBS2 & CTGF & SFRP2 & INHBA & THBS2 & SPOCK1 \\
\hline 12 & MGP & CTGF & STEAPI & EFEMP1 & COL10A1 C & COL10A1 & | RARRESI & STEAP1 & COL8A1 & CTSK & CYPIBI & SERPINE2 & MATN3 & $\mathrm{BNC2}$ & EFEMP1 & FBLN1 & COL8A1 & CTHRC1 & LUM & COL12A1 & ADM \\
\hline 13 & DCN & SFRP2 & MMP2 & OMD & COL8A1 & CTHRC1 & CIS & COL1A2 & FBLN1 & CCDC80 & COLIA1 & FBLN1 & MEG3 & MRC2 & MRC2 & VCAN & THBS2 & MMP2 & FBLN1 & HTRA1 & MMP2 \\
\hline 14 & CYPIB1 & $\mathrm{CIS}$ & CYPIBI & RP11-572Cl & PDPN & CCDC80 & VCAN & PTGDS & OMD & ISLR & APOD & SCN7A & ISLR & DPYSL3 & COL6A3 & CCDC80 & MMP2 & COL10A1 & MMP2 2 & MMP14 & ARL4C \\
\hline 15 & COLIA2 & OMD & PTGDS & CCDC80 & CXCL14 S & SFRP4 & PTGDS & ISLR & THBS2 & C3 & CLDN11 & LTBP2 & FBLN1 & COL1A2 & PDPN & COL1A1 & & & COL6A3 & SFRP2 & MEG3 \\
\hline 16 & MOXD1 & SPON2 & FBLN1 & IGFI & MMP23B & CTSK & $\mathrm{ClR}$ & LXN & FAP & EFEMP1 & CTSK & APOD & $\mathrm{COL} 1$ & FBLN5 & VCAN & S100A6 & INHBA & AEBP1 & OMD & SULF1 & GJA1 \\
\hline 17 & PTGDS & C3 & RARRES & 1 TSHZ2 & ABCA9 & COL3A1 & CTSK & OLFML3 & MFAP2 & MGP & FBLN1 & PTGDS & COL3A1 & CREB3L1 & DPYSL3 & COL8A1 & CIQTNF3 & $\mathrm{COL} 12 \mathrm{~A} 1$ & MEG3 & LUM & VCAN \\
\hline 18 & FBLN5 & $\mathrm{F} 2 \mathrm{R}$ & DCN & ITM2A & LUM & S100A10 & PDGFR & THBS2 & COL6A3 & TSHZ2 & MMP2 & ISLR & SFRP4 & COL3A1 & LOX & TMSB 10 & MATN3 & DCN & COL8A1 & SDC1 & FIBIN \\
\hline 19 & PDGFRA & ANKH & COL3A1 & SFRP4 & PDGFRL & THBS2 & SERPINF1 & FBLN1 & RARRES1 & 1 PDGFRA & COL3A1 & PODN & CXCL14 & OMD & GJA1 & ClS & SFRP4 & SPARC & ISLR & DCN & COL1A2 \\
\hline 20 & COL6A3 & CTSK & MFAP5 & RARRES1 & STXBP6 & HTRA1 & MOXD1 & MGST1 & EFEMP1 & OMD & PTGDS & DCN & ITGBL1 & RARRES1 & APOD & COL1A2 & HTRA1 & TMSB10 & MMP11 & MFAP5 & ZFHX4 \\
\hline 21 & CTHRC1 & CIR & FBLN5 & COL8A1 & SVEP1 & SEMA3C & GPNMB & MEG3 & COL1A2 & FBLN5 & FBLN2 & MMP2 & COL6A3 & PODN & FAP & COL3A1 & ITGBL1 & MMP14 & MFAP5 & VCAN & MFAP2 \\
\hline 22 & FAP & IGFBP3 & DL1A1 & OGN & & FBLN2 & RARRES2 & PDPN & AN & & C. & MC & & LSAMP & MX & HTRA1 & MFAP5 & SDC1 & PPAPDCI & COL6A3 & MME \\
\hline 23 & $\mathrm{~F} 2 \mathrm{R}$ & MOXD1 & ISLR & $\mathrm{C} 7$ & ABCA6 & LRPI & FBLN5 & MFAP2 & FNDC1 & OGN & C3 & C7 & IGFL2 & THBS2 & PODN & CD99 & CXCL14 & POSTN & CTSK & GJB2 & MFAP5 \\
\hline 24 & ISLR & CTHRCI & c & DPT & MFAP2 & MRC2 & ISLR & DPT & SPON2 & SERPINF & $\mathrm{x}$ & MGS & RARRES2 & 2SVEPI & CXCL14 & ISLR & GJB2 & FBLN1 & MXRA5 & GREM1 & RAB3B \\
\hline 25 & TIMP1 & MEG3 & MEG3 & BICCI & BNC2 & PDPN & ITGBLI & C3 & PDPN & CTGF & RARRES1 & SPOCK1 & FNDCl & ITGBL1 & COL8A1 & SERPINFI & IGFBP3 I & INHBA & FNDC1 & TIMP2 & ITGBL1 \\
\hline 26 & C7 & PDGFRA & CTHRC1 & CTHRC1 & WNTSA & MXRA5 & COL10A1 & MRC2 & CTSK & FBLN2 & RP11-572C15. & $6 \mathrm{CCDC} 80$ & COL5A1 & PTGDS & SFRP4 & TSC22D3 & $\mathrm{CCDC} 80 \mathrm{~S}$ & SERPINH 1 & ICOLIAI & COL5A2 & GJB2 \\
\hline 27 & PHLDA3 & FBLN5 & MOXD1 & PODN & CST3 & OMD & CYPIBI & PTGIS & MFAP5 & COL6A3 & THBS2 & SLC19A2 & OMD & FAP & SEMA3C & FTL & CD99 & MXRA5 & SDCl & FAP & COL3A1 \\
\hline 28 & OMD & ITM2A & COL6A3 & COL6A3 & SFRP2 & ITGBL1 & C7 & MOXD1 & HTRA3 & PODN & C7 & HTRA3 & CST4 & LUM & LRP1 & MFAP2 & CIS & HTRA1 & VCAN & MFAP2 & NTM \\
\hline 29 & FBLN2 & COLIA2 & MGP & CXCL14 & MOXD1 I & FBN1 & PLXDC2 & HSDl1B1 & LRP1 & LTBP2 & LAMA2 & MOXD1 & INHBA & FBLN1 & NTM & ANXA2 & LOXL1 & MMP11 & GREM1 & CTSK & PDLIM4 \\
\hline 30 & SCN7A & PTGDS & C7 & COL1A2 & ZFHX4 & RARRES2 & 2 CTHRCI & SFRP4 & COLIA1 & ITGBLI & OLFML3 & ITGBLI & GJB2 & SULF1 & C3 & LAPTM4A & FIBIN & ISLR & PDPN & COL5A1 & CMTM8 \\
\hline 31 & EFEMP1 & RARRESI & CILP & ISLR & RARRES1 I & PLXDC2 & DPT & LSAMP & DIO2 & C7 & MFAP4 & CTS & IGFBP3 & INHBA & & NNMT & FBLN1 & MEG3 & FBLN2 & PPAPDCIA & \\
\hline 32 & COLI0AI & OLFML3 & COL8A1 & MEG3 & BOC & CXCL14 & CLU & PLXDC2 & DCN & PLXDC2 & SLIT2 & FBLN2 & HTRA3 & SEMA3C & UNC5B & ClR & PALLD & TIMP2 & MFAP2 & $\mathrm{FN1}$ & NTSE \\
\hline 33 & SERPINF1 & IOGN & THBS2 & CTSK & PODN & PDGFRA & NPC2 & MFAP4 & ALDH IA 3 & 3MOXDI & IGFI & CTHRCl & CST1 & LOX & LOXL1 & RPL27A & $\mathrm{SDCl}$ & FSTL1 & CIQTNF3 & TMEM158 & TENM3 \\
\hline 34 & $\mathrm{BNC2}$ & ITGBLI & MRC2 & HSD11B1 & TMEM1 19 N & MATN3 & MEG3 & OGN & LOX & TMEM119 & LRP1 & SVEP1 & FBLN2 & PDPN & COL5A2 & INHBA & MFAP2 & COL6A2 & COL5A2 & POSTN & EPDR1 \\
\hline 35 & CTSK & PTCH1 & NR2F1 & CTGF & PTGIS & COL8A1 & RP11-572Cl & $\mathrm{COL}_{6 \mathrm{~A} 3}$ & FBLN2 & COL8A1 & PDLIM3 & MXRA5 & MFAP5 & IGF1 & SCARA3 & NUPR1 & ANXA2 & CTSK & CDH11 & ANTXR1 & MYHIO \\
\hline 36 & MRC2 & COL8A1 & ITGBLI & CIS & OGN & NBL1 & MGP & SPON2 & OLFML3 & LRP1 & LTBP2 & STEAP1 & MXRA5 & LAMP5 & COL8A2 & LGALS1 & APOD & MFAP2 & LOX & PLAU & LOX \\
\hline 37 & TSHZ2 & $\mathrm{BOC}$ & TSHZ2 & COL10A1 & FAP & TMEM119 & $9 \mathrm{COL} 6 \mathrm{~A} 3$ & COLIA1 & TMEM119 & 9 RARRES1 & FBLN5 & NEGR1 & THBS2 & FBLN2 & PTGDS & COL6A3 & OMD & MFAP5 & COL3A1 & INHBA & COL8A1 \\
\hline 38 & LRPI & SERPINF 1 & ISTEAP2 & SERPI & EFEMP1 & HTRA3 & LTBP2 & LTBP2 & MEG3 & BOC & MGP & C3 & CTGF & OGN & CCDC80 & & ISLR & GAS1 & PDGFRA & GJA1 & EVAlA \\
\hline 39 & SLIT2 & MGP & MFAP2 & SERPINFI & LAMA2 & FAP & AEBP1 & PODN & FGF7 & CYBRD1 & MEG3 & RARRESI & MRC2 & PTGFRN & ALDH $1 A 3$ & $3 \mathrm{OMD}$ & SLC6A6 & LRRC15 & F13A1 & LGALS1 & MXRA5 \\
\hline 40 & COLIA1 & IGFBP6 & PDGFRL & LTBP2 & GSTI & MEG3 & PDPN & DCN & TMSB 10 & LX & BIC & RPII & $6 \mathrm{GJA1}$ & GAS7 & FGFRI & CD: & & $\mathrm{BA} 2$ & FRMD6 & PTH & BIC \\
\hline 41 & SVEPI & TIMP1 & PDPN & NEGRI & IGF1 & FNDCl & COLIA1 & CTSK & CXCL14 & LOX & MRC2 & PLXDC2 & FAP & CXCL14 & BOC & PDPN & COL1A2 & FBLN2 & APOD & CD 99 & Clorf198 \\
\hline 42 & THBS2 & MFAP4 & PLXDC2 & & $\mathrm{F} 2$ & & & SPOCK1 & ITGBLI & HTRA & & & N2 & & PLXDC2 2 & LOXL1 & MMP11 & GREM1 & COL1A2 & NBL1 & \\
\hline 43 & TMEM119 & CLEC11A & OMD & $\mathrm{CIR}$ & F3 & DPYSL3 & S100A13 & COL3AI & IGFBP3 & RP11-572 & SABL2 & ABI3BP & BICC1 & FNDCl & MFAP2 & FIBIN & MEG3 & APOD & DIO2 & NTM & PDGFC \\
\hline 44 & IGFBP3 & INHBA & MMP23B & FGF7 & MMP2 & SLC6A6 & $\mathrm{OG}$ & RARRESI & $1 \mathrm{CDH} 11$ & CYP1B1 & MXRA5 & FAP & EMP1 & HTRA3 & MFAP5 & NTM & PLXDC2 & COL8A1 & CD55 & RARRES2 & FBLN2 \\
\hline 45 & $\mathrm{C} 1 \mathrm{~S}$ & MXRA8 & LXN & & SFI & & & SVEPI & IGI & & & & Ft & & B 10 & & & & HTRA1 & FBLN1 & B4GALT1 \\
\hline
\end{tabular}


2.1.3 Dominant fibroblastic population in the normal samples. Table 4 shows the attractor metagenes of the 11 normal samples by listing the 20 top-ranked genes for each sample, in which commonly shared genes are color-coded. There is a striking similarity among them, indicating that they represent a stable and normally occurring cell population. Specifically, there are 18 genes shared among the top 50 genes in the attractors of at least ten of the eleven normal samples, listed by average rank $\left(P<10^{-7}\right.$ by permutation test). In addition to fibroblastic markers, there are several strongly expressed adipose-related or stemness-related genes in the list, such as APOD, CFD, CXCL12 and DPT, revealing that they are ASCs. Consistently, Gene Set Enrichment Analysis of these 18 genes identified most significant enrichment $\left(\right.$ FDR $q$ value $\left.=5.38 \times 10^{-29}\right)$ in the "BOQUEST_STEM_CELL_UP" dataset of genes upregulated in stromal stem cells from adipose tissue versus the non-stem counterparts [22].

Table 4. Top 20 genes of the identified attractors for each pancreatic normal sample (N1-N11).

\begin{tabular}{|c|c|c|c|c|c|c|c|c|c|c|c|}
\hline Rank & N1 & N2 & N3 & $\mathrm{N} 4$ & N5 & N6 & N7 & N8 & N9 & N10 & N11 \\
\hline 1 & DCN & LUM & LUM & $\mathrm{C} 7$ & APOD & LUM & PTGDS & $\mathrm{C} 7$ & $\mathrm{DCN}$ & MMP2 & LUM \\
\hline 2 & LUM & DCN & FBLN1 & FBLN5 & DPT & DCN & APOD & LUM & LUM & APOD & DCN \\
\hline 3 & $\mathrm{C} 7$ & $\mathrm{C} 7$ & $\mathrm{C} 7$ & LUM & FBLN5 & FBLN1 & LUM & DCN & $\mathrm{C} 7$ & LUM & FBLN1 \\
\hline 4 & FBLN1 & FBLN1 & PTGDS & DCN & PDGFRA & ADH1B & FBLN1 & APOD & FBLN1 & EFEMP1 & SFRP2 \\
\hline 5 & MGP & APOD & C1S & APOD & CXCL12 & DPT & $\mathrm{C} 7$ & FBLN1 & APOD & CTSK & CFD \\
\hline 6 & $\mathrm{C} 1 \mathrm{~S}$ & MGP & DPT & PTGDS & LUM & ABCA8 & ADH1B & SFRP2 & SFRP2 & SFRP2 & APOD \\
\hline 7 & CCDC80 & C1S & PDGFRA & FBLN1 & COL6A3 & $\mathrm{C} 3$ & DPT & PTGDS & SERPINF1 & PLTP & MGP \\
\hline 8 & PTGDS & DPT & APOD & $\mathrm{ClR}$ & PTGDS & APOD & COL6A3 & $\mathrm{CCDC} 80$ & PTGDS & MGST1 & SERPINF1 \\
\hline 9 & DPT & CCDC80 & SFRP2 & DPT & $\mathrm{C} 7$ & MMP2 & EFEMP1 & FBLN5 & GSN & LSP1 & CCDC80 \\
\hline 10 & $\mathrm{C} 1 \mathrm{R}$ & PTGDS & $\mathrm{DCN}$ & SRPX & CCDC 80 & C1S & PDGFRA & $\mathrm{C} 1 \mathrm{~S}$ & $\mathrm{C} 1 \mathrm{~S}$ & FBLN1 & $\mathrm{C} 3$ \\
\hline 11 & APOD & FBLN5 & CXCL12 & FMO2 & CFD & $\mathrm{C} 7$ & CXCL12 & CXCL12 & SEPP1 & SPON2 & ADH1B \\
\hline 12 & SEPP1 & SEPP1 & $\mathrm{ClR}$ & SEPP1 & MRC2 & PTGDS & SCN7A & $\mathrm{C} 3$ & CCDC80 & PTGDS & PTGDS \\
\hline 13 & FBLN5 & COL1A2 & COL6A3 & CXCL12 & FGF7 & SFRP2 & MMP2 & CFD & DPT & SVEP1 & $\mathrm{C} 7$ \\
\hline 14 & CXCL12 & SFRP2 & ADH1B & CYR61 & SFRP2 & FBLN5 & MEG3 & $\mathrm{C} 1 \mathrm{R}$ & OLFML3 & CXCL12 & C1S \\
\hline 15 & EFEMP1 & SRPX & SPON2 & SFRP2 & MARCKS & $\mathrm{C} 1 \mathrm{R}$ & C1S & MGP & FBLN5 & SCN7A & CST3 \\
\hline 16 & COL1A2 & SERPINF1 & CFD & CLEC11A & LRP1 & CXCL12 & OLFML3 & $\mathrm{CFH}$ & $\mathrm{ClR}$ & COL6A3 & $\mathrm{C} 1 \mathrm{R}$ \\
\hline 17 & SFRP2 & OLFML3 & LAMA2 & PDGFRA & FMO2 & CST3 & SVEP1 & COL6A3 & PTN & CCDC80 & CXCL14 \\
\hline 18 & ALDH1A1 & CST3 & $\mathrm{C} 3$ & NR2F1 & NR2F1 & MGP & $\mathrm{DCN}$ & SRPX & MGP & COLEC11 & MMP2 \\
\hline 19 & CFD & MEG3 & FBLN5 & C1S & TNXB & CCDC 80 & SFRP2 & EFEMP1 & ALDH1A1 & PDGFRA & GPNMB \\
\hline 20 & COL6A3 & $\mathrm{C} 1 \mathrm{R}$ & ABCA8 & ABCA8 & DCN & MRC2 & MRC2 & SEPP1 & PDGFRA & HBP1 & S100A4 \\
\hline
\end{tabular}


To investigate the nature of this ASC population, we referred to recent results from single-cell analysis of general human adipose tissue [23]. We used the dataset with the single-cell expression profiles of all 26,350 cells taken from the SVF of normal adipose tissue from 25 samples. The first column in Table 5 contains the top 30 ranked genes in the corresponding attractor signature. The second column contains the top 30 ranked genes of the consensus attractor (Materials and Methods) of the 11 normal pancreatic samples, representing the main state of the normal fibroblastic population. There are 14 overlapping genes in the two lists $\left(P=10^{-37}\right)$, and most of the non-highlighted genes in each column are still ranked highly in the other column. This extreme similarity of the two gene expression profiles indicates that they correspond to the same naturally occurring cell population.

Table 5. Comparison of the attractors (top 30 genes) identified in the SVF of normal adipose tissue (Dataset 1 ) and in the normal pancreatic samples (Dataset 2). Common genes are highlighted in yellow.

\begin{tabular}{|l|ll|l|ll|}
\hline Rank & Dataset 1 & Dataset 2 & Rank (cont'd) & Dataset 1 & Dataset 2 \\
\hline $\mathbf{1}$ & DCN & LUM & $\mathbf{1 6}$ & FOS & PDGFRA \\
$\mathbf{2}$ & LUM & DCN & $\mathbf{1 7}$ & MGST1 & SRPX \\
$\mathbf{3}$ & APOD & FBLN1 & $\mathbf{1 8}$ & COL1A2 & COL6A3 \\
$\mathbf{4}$ & CFD & C7 & $\mathbf{1 9}$ & COL6A3 & ADH1B \\
$\mathbf{5}$ & CXCL14 & APOD & $\mathbf{2 0}$ & LAPTM4A & CFD \\
$\mathbf{6}$ & MGP & PTGDS & $\mathbf{2 1}$ & CXCL12 & OLFLM3 \\
$\mathbf{7}$ & SERPINF1 & SFRP2 & $\mathbf{2 2}$ & WISP2 & SERPINF1 \\
$\mathbf{8}$ & GSN & C1S & $\mathbf{2 3}$ & SRPX & MMP2 \\
$\mathbf{9}$ & GPX3 & CCDC80 & $\mathbf{2 4}$ & JUN & CST3 \\
$\mathbf{1 0}$ & MFAP4 & MGP & $\mathbf{2 5}$ & MMP2 & SEPP1 \\
$\mathbf{1 1}$ & PLAC9 & DPT & $\mathbf{2 6}$ & COL6A2 & ABCA8 \\
$\mathbf{1 2}$ & S100A13 & CXCL12 & $\mathbf{2 7}$ & C1S & COL1A2 \\
$\mathbf{1 3}$ & IGFBP6 & C1R & $\mathbf{2 8}$ & CCDC80 & LAMB1 \\
$\mathbf{1 4}$ & DPT & FBLN5 & $\mathbf{2 9}$ & EGR1 & SVEP1 \\
$\mathbf{1 5}$ & MFAP5 & C3 & $\mathbf{3 0}$ & PCOLCE & MEG3 \\
\hline
\end{tabular}


2.1.4 Further demonstration of the continuity of the transition. As an additional confirmation of the continuity of the transition (as opposed to the presence of a mixture of distinct fibroblastic subtypes), Fig 1 shows scatter plots, color-coded for the expression of fibroblastic marker $L U M$, for the fibroblast-rich samples T11 and T23, demonstrating the presence in each sample of cells representing the full range of the continuously varying transition from APOD to COL11A1expressing cells.

Fig 1. Scatter plots for fibroblast-rich samples for patients T11 and T23.
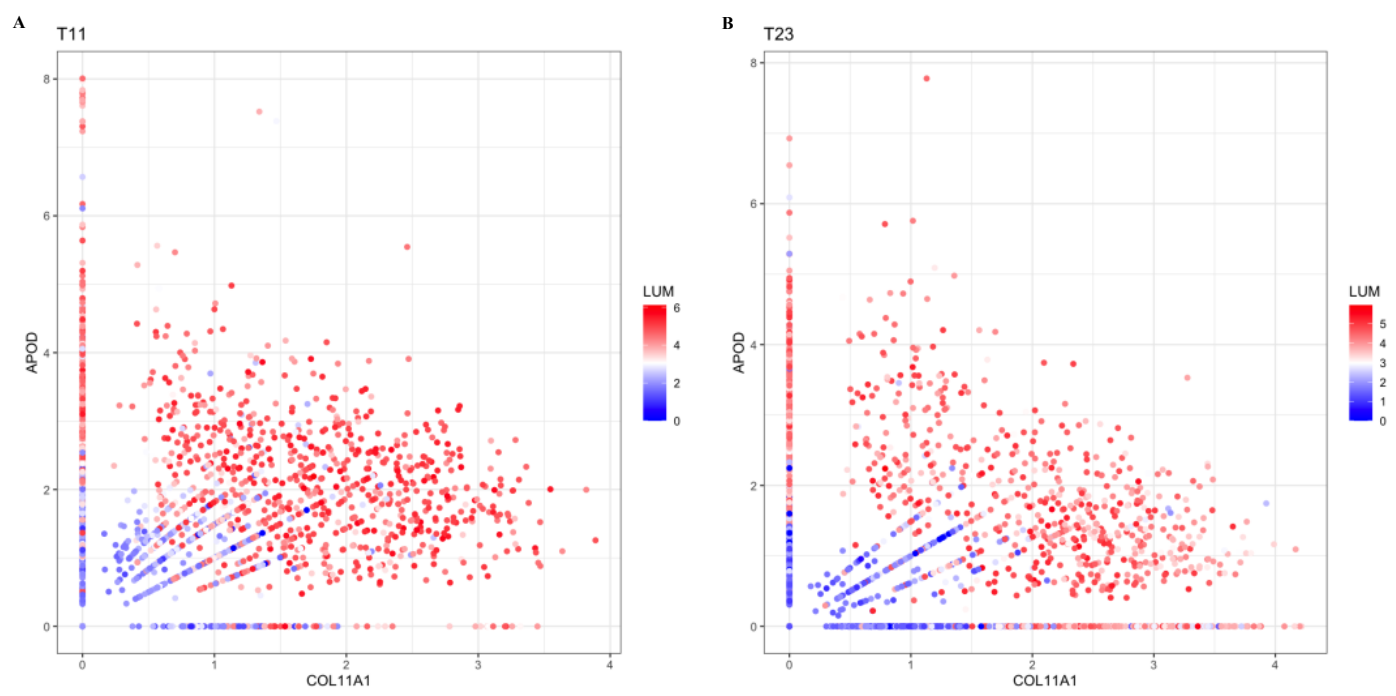

To further investigate the continuous transition, we partitioned the 34 pancreatic samples into three groups, considering whether the corresponding attractor includes COL11A1 among the top 100 genes (S1 Table). Group 1 includes the eleven normal samples (N1 to N11). Group 2 includes twelve tumor samples in which COL11A1 is not included (T1, T2, T3, T5, T10, T12, T13, T14, T16, T18, T19, T24). Group 3 includes eleven cancer samples in which it is included (T4, T6, T7, T8, T9, T11, T15, T17, T21, T22, T23). We then applied the consensus version of the attractor finding algorithm (Materials and Methods) and identified the signatures representing the main state of the fibroblasts for each of the above three sample groups (Table 6), so we can observe the genes whose ranking changes significantly during the transition. 
Table 6. Top 50 genes of the consensus attractors for three different PDAC sample groups. Group1: normal samples; Group 2: tumor samples in which the dominant fibroblastic population does not include COL11A1; Group 3: tumor samples in which the dominant fibroblastic population includes COL11A1.

\begin{tabular}{|c|c|c|c|c|c|c|c|}
\hline Rank & Group1 & Group2 & Group3 & Rank (cont'd) & Group1 & Group2 & Group3 \\
\hline 1 & LUM & LUM & COL1A1 & 26 & ABCA8 & PLXDC2 & NTM \\
\hline 2 & DCN & $\mathrm{DCN}$ & COL10A1 & 27 & COL1A2 & COL1A1 & NBL1 \\
\hline 3 & FBLN1 & APOD & COL1A2 & 28 & LAMB1 & OGN & ITGBL1 \\
\hline 4 & $\mathrm{C} 7$ & SFRP4 & CTHRC1 & 29 & SVEP1 & C1S & FN1 \\
\hline 5 & APOD & SFRP2 & SFRP2 & 30 & MEG3 & PTGDS & MFAP2 \\
\hline 6 & PTGDS & VCAN & COL3A1 & 31 & PCOLCE & ITGBL1 & RARRES2 \\
\hline 7 & SFRP2 & FBLN1 & MMP2 & 32 & NEGR1 & TSHZ2 & GJB2 \\
\hline 8 & $\mathrm{C} 1 \mathrm{~S}$ & MMP2 & LUM & 33 & FGF7 & PODN & MXRA5 \\
\hline 9 & CCDC80 & PDGFRA & VCAN & 34 & LRP1 & SERPINF1 & LOX \\
\hline 10 & MGP & EFEMP1 & THBS2 & 35 & CYR61 & OMD & MEG3 \\
\hline 11 & DPT & RARRES1 & COL6A3 & 36 & ALDH1A1 & COL10A1 & MRC2 \\
\hline 12 & CXCL12 & FBLN5 & $\mathrm{DCN}$ & 37 & EFEMP1 & HSD11B1 & HTRA1 \\
\hline 13 & $\mathrm{C} 1 \mathrm{R}$ & ISLR & COL11A1 & 38 & FMO2 & IGF1 & PDPN \\
\hline 14 & FBLN5 & COL6A3 & APOD & 39 & $\mathrm{CFH}$ & RP11-572C15.6 & FBLN2 \\
\hline 15 & $\mathrm{C} 3$ & $\mathrm{C} 3$ & SFRP4 & 40 & PLTP & LTBP2 & C1QTNF3 \\
\hline 16 & PDGFRA & CCDC80 & COL5A2 & 41 & SLIT2 & FBLN2 & TMSB10 \\
\hline 17 & SRPX & CTSK & FAP & 42 & MRC2 & COL3A1 & POSTN \\
\hline 18 & COL6A3 & CTHRC1 & FBLN1 & 43 & SCN7A & STEAP1 & CDH11 \\
\hline 19 & ADH1B & CYP1B1 & COL8A1 & 44 & LAMA2 & LXN & MMP14 \\
\hline 20 & CFD & MEG3 & COL5A1 & 45 & TIMP1 & $\mathrm{BOC}$ & CCDC80 \\
\hline 21 & OLFML3 & COL1A2 & AEBP1 & 46 & COL6A2 & MRC2 & SULF1 \\
\hline 22 & SERPINF1 & MOXD1 & MFAP5 & 47 & CXCL14 & LRP1 & TMEM119 \\
\hline 23 & MMP2 & $\mathrm{C} 7$ & INHBA & 48 & LTBP4 & THBS2 & CXCL14 \\
\hline 24 & CST3 & MGP & CTSK & 49 & EMP1 & MFAP4 & FNDC1 \\
\hline 25 & SEPP1 & COL8A1 & ISLR & 50 & GSN & FAP & IGFBP3 \\
\hline
\end{tabular}

Although there are many shared genes, the groups have distinct gene rankings. Group 1 (normal samples) contains many adipose-related genes, consistent with Table 4. Group 3 contains, in addition to COL11A1, many among the other CAF genes, such as THBS2, INHBA, MMP11, AEBP1, MFAP5, COL10A1 and VCAN.

Group 2 displays an intermediate state, including markers of both ASCs as well as CAFs. To find the most unique and representative gene(s) for Group 2, we focused on the top 20 genes of its attractor and calculated the ranking increment when compared with the rankings of the same genes in the attractors of the other two groups. We considered a ranking increase of greater than 50 as significant. As a result, we found that RARRES1 is the only gene satisfying the criterion in both comparisons. 
Gene RARRES1 (aka TIG1) appears in the beginning of the ASC to COL11A1-expressing CAF transition process and in all samples of Group 2. This is consistent with the known fact that it plays an important, though not yet clear, role in regulating proliferation and differentiation of adipose tissue derived mesenchymal stem cells [24]. On the other hand, the expression of RARRES1 is reduced in Group 3, disappearing completely in the final stages of differentiation when COL11A1 is expressed strongly (consistent with suggestions that it is a tumor suppressor $[25,26]$ ).

We also performed differential expression (DE) analysis on the classified fibroblasts between the three sample groups defined above (Materials and Methods; see complete DE gene lists in S2 Table). Importantly, the results of such analysis represent the full population of fibroblasts and not necessarily the expression changes in the particular cells undergoing the ASC to COL11A1expressing CAF transition.

For the 'Group1 to Group 2' stage, genes $C F D$ and $D P T$ are the most downregulated genes, consistent with the downregulation of adipose-related genes. On the other hand, the top upregulated gene is phospholipase A2 group IIA $(P L A 2 G 2 A)$, whose encoded protein is a member of a family of enzymes catalyzing the hydrolysis of phospholipids into free fatty acid. At the same time, other extracellular matrix genes are also strongly upregulated, as expected.

For the 'Group 2 to Group 3' stage, remarkably PLA2G2A again shows as the top DE gene, however this time it is down-regulated. Similarly, the tumor suppressor RARRES1, which was significantly up-regulated in the early stage becomes down-regulated in this stage. On the other hand, the most significantly upregulated genes, following the top gene COL11A1, were matrix metalloproteinase (MMP11), collagens of type X and XII (COL1OA1 and COL12A1), thrombospondin 2 (THBS2), growth factor binding protein 3 (IGFBP3), while CFD, APOD and 
C7 continued decreasing their expression levels. Thus, this stage represents the final formation of COL11A1-expressing CAFs.

The top differentially expressed gene $P L A 2 G 2 A$ is not among the top genes of any attractors we identified and is expressed by less than half of cells, even in Group 2 in which it appears. Therefore, it is expressed by cells in Group 2 that are not among the ones undergoing the ASC to COL11A1expressing CAF transition, although it probably still plays an important related role and many previous studies referred to its effects on prognosis of multiple cancer types [27-29]. This gene is a phospholipase catalyzing the hydrolysis of phospholipids into fatty acids. On the other hand, it has been recognized that fatty acid oxidation is associated with the final COL11A1-expressing stage of the transition [30]. These results suggest that lipid metabolic reprogramming plays an important role in the metastasis-associated biological mechanism [31], providing energy for the metastasizing tumor cells.

2.1.5 Trajectory Inference. We independently applied trajectory inference analysis on the PDAC fibroblasts by using the Slingshot [32] method. We first performed unsupervised clustering on the identified fibroblasts (Materials and Methods), resulting in four subgroups X1, X2, X3, X4 (S1A Fig) with the top differentially expressed genes shown in S1B Fig. One of these clusters (X4) was discarded from further TI analysis, because it mainly expressed the IL1 CAF marker HAS1 (Hyaluronan Synthase 1), which is not expressed by either ASCs or COL11A1-expressing CAFs (and does not appear at all in S1 Table), and contained only 3\% of fibroblasts resulting almost exclusively from patient T11 (S1C Fig).

As seen from the list of top differentially expressed genes, X1 contains CAF genes top ranked (including MMP11, COL11A1, THBS2, INHBA), X2 has RARRES1 at the top, and X3 has ASC genes top ranked, including DPT, C7, CXCL12 and CFD. Consistently, S2A Fig and S2B Fig show 
the single trajectory path resulting from TI analysis, where $\mathrm{X} 3$ is the starting point and $\mathrm{X} 1$ is the end point of the trajectory, while $\mathrm{X} 2$ (highly expressing RARRES1), is an intermediate point, thus validating the continuous ASC to COL11A1-expressing CAF transition. S3 Table shows the top 100 genes with zero $P$ value, ranked by their variances, resulting from pseudotime-based differential gene expression analysis (Materials and Methods). We can clearly identify as topranked several ASC genes, as well as CAF genes, while some general fibroblastic markers, such as $D C N$, are missing, consistent with the continuity of the ASC to COL11A1-expressing CAF transition. We then used a generalized additive model (GAM) fit to pseudotime-ordered expression data to visualize the trend of gene expressions (Fig 2A).

Fig. 2. Trajectory analysis of PDAC. A. GAM fit to pseudotime ordered expression data to visualize the trend of gene expressions. B. Expression of adipose-related genes along the transition lineage. The x axis shows the cell orders and the $\mathrm{y}$ axis shows the normalized read count. C. Expression of COL11A1-associated genes along the transition lineage. D. Expression of RARRES1 and SFRP4 genes along the transition lineage.

A

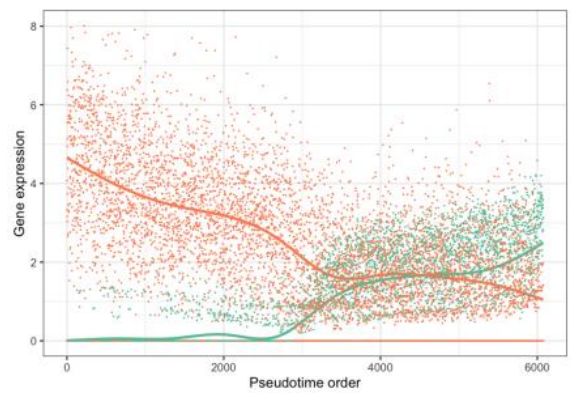

C

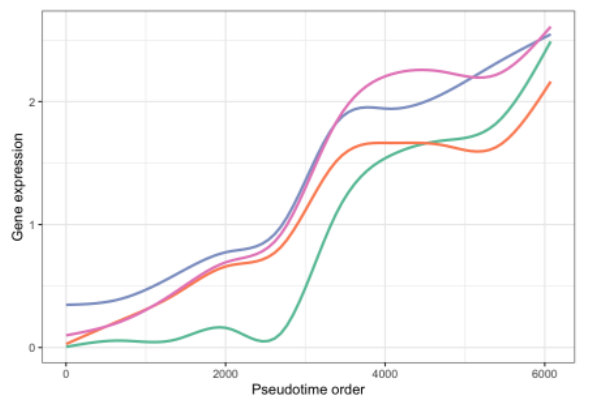

B

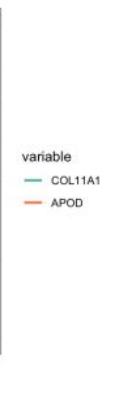

D

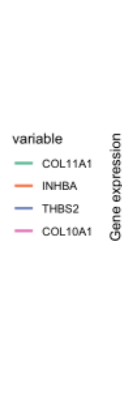

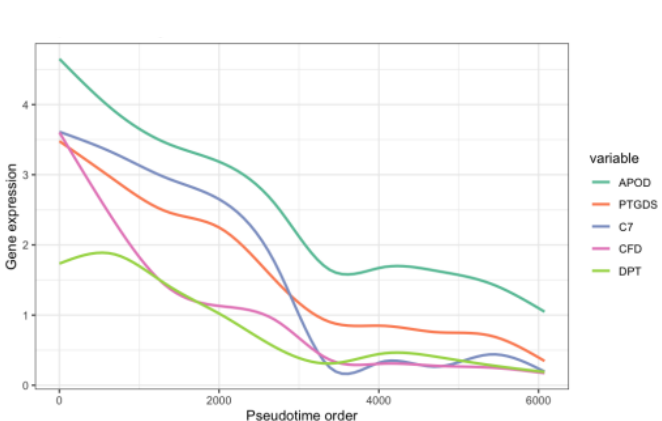

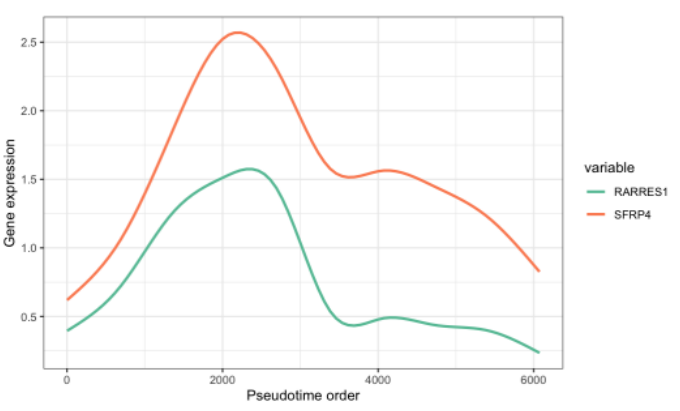


There was a prominent difference between adipose-related genes and COL11A1-associated genes. The expression of the adipose-related genes steadily fell across the process (Fig 2B), while the expression of COL11A1-associated genes gradually increased (Fig 2C). There is a significant negative correlation between these two groups of genes, e.g., COL11A1 (the last among those genes to increase its expression) was exclusively overexpressed in the mature CAFs, which did not express C7. Of particular interest, genes RARRES1 and SFRP4 (Fig 2D) increased consistently at the beginning and then decreased after reaching a peak, suggesting that they play important roles in the differentiation path.

\subsection{Validation in other cancer types}

Next, we validated the ASC to COL11A1-expressing CAF transition in other solid cancer types. Although we could not find currently available datasets as rich as the PDAC dataset, we selected those containing a large (at least 100) number of fibroblasts and separately analyzed each of them, obtaining consistent results. Specifically, we used four scRNA-seq datasets from head and neck cancer (HNSCC) [33], ovarian cancer [34], lung cancer [35] and breast cancer [36].

The COL11A1-expressing CAF signature has been confirmed to be a pan-cancer signature [3739]. Therefore, the most important validation task would be to confirm the existence of the APOD/CFD/CXCL12/MGP/PTGDS-expressing ASCs as the starting point of the transition, and to also confirm that some samples are at an intermediate stage, expressing genes such as RARRES1, SFRP4 and THBS2, in addition to the core ASC genes, demonstrating that they are at an intermediate stage of the transition.

2.2.1 Head and neck squamous cell carcinoma. For the HNSCC dataset, the authors of the paper presenting the data [33] reported that the cancer-associated fibroblasts in the dataset can be 
partitioned into two subsets, which they name CAF1 and CAF2. In table s5 of that paper, the top three differentially expressed genes of the CAF2 group are CFD, APOD and CXCL12, while the full gene list for CAF2 presented in the same table s5 also includes genes $M G P, C 3, C 7, D P T$, PTGDS. This strongly suggests that the partitioning used in the paper was influenced by the presence of an ASC cell subpopulation, identical, or at least very similar to, those discovered in the PDAC. Similarly, the list of differentially expressed genes for CAF1 in table s5 includes genes INHBA, THBS2, CTHRC1, POSTN, MMP11, COL5A2, COL12A1, suggesting that the identified CAF1 subpopulation was influenced by the presence of differentiated CAFs, which would eventually express COL11A1. Finally, gene RARRES1 also appears among the list of CAF2 genes, suggesting that it was captured among cells which had started the process of ASC to COL11A1-expressing CAF transition.

In our independent analysis, we performed clustering identifying 1,026 fibroblasts from all available cells (S3A Fig; Materials and Methods). There were two fibroblastic clusters (X7 and X9) expressing CAF associated genes (COL11A1, COL12A1, MMP11, INHBA, THBS2, COL10A1, COL8A1, FN1) and ASC associated genes (APOD, C7, PTGDS), respectively (S4 Table), which confirmed the presence of these two populations in HNSCC.

Among the individual patients, we found that the most prominent case is sample HNSCC28, which contains a rich set of cells undergoing differentiation. Applying the attractor finding algorithm on the fibroblasts of that sample (S5 Table) resulted in genes LUM, APOD, COL6A3, PDGFRA, DCN, and CFD being among the top-ranked, revealing that it represents an ASC population. Furthermore, the presence of genes THBS2, MFAP5 and VCAN in the same attractor reveals that these cells have already started undergoing the transition. 
2.2.2 Ovarian cancer. For the ovarian dataset, the clustering results showed two clusters (X6 and X9) expressing COL11A1-associated genes and ASC-associated genes, respectively (S3B Fig, S6 Table; Materials and Methods). Among the individual patients, we found that the ones validating our hypotheses most are HG2F and LG2, both of whose datasets, consistently, contain cells from the fatty omental tissue. S5 Table includes the corresponding two attractors identified in the cells of each patient. Among the top ranked genes for $\mathrm{HG} 2 \mathrm{~F}$ are $D C N, L U M, C 1 S, C 7$, and $C 3$, but also RARRES1, suggesting that they represent fibroblasts undergoing the transition, while the LG2-based attractor contains highly ranked all three genes COL11A1, INHBA, THBS2.

2.2.3 Lung cancer. The dataset contains a large number $(>50,000)$ of cells, but we only classified $\sim 2 \%(=1,346)$ among them as mesenchymal cells, including fibroblasts and pericytes (Materials and Methods). Among those cells, there were two fibroblastic clusters (X1 and X2) expressing related genes (COL11A1, COL12A1, MMP11, INHBA, THBS2, COL10A1, COL8A1, FN1) and ASC related genes (APOD, C7, PTGDS), respectively (S3C Fig, S7 Table). The presence of the transition is evident by the attractors identified in the mesenchymal cells for patients 4 and 3 (S5 Table). The former prominently contains genes $C F D, P T G D S$ and $C 7$, while the latter contains THBS2, COL1OA1 and INHBA.

2.2.4 Breast cancer. The size of the breast cancer dataset is small ( 1,500 cells in total), and 169 cells among them were classified as mesenchymal (Materials and Methods). By further clustering these cells, we identified ASCs (X1) and COL11A1-expressing CAFs (X3) (S3D Fig, S8 Table). ASC related genes $(A P O D, M F A P 4, C F D)$ were identified in X1, while CAF-related genes (COL10A1, COL11A1, MMP11, INHBA, FN1, THBS2, AEBP1, COL12A1) are among the top 15 of X3. Patients PT089 and PT039 contain the highest proportions (>50\%) of the ASC and 
COL11A1-expressing CAF subpopulations, respectively, and we found consistent results in their attractors (S5 Table), as the former contains C1S, C1R, CXCL12, PTGDS, C3, while the latter contains THBS2, COL11A1, COL10A1, at top-ranked positions.

\subsection{Potential therapeutic targets}

This work provides opportunities for identifying therapeutic targets inhibiting the cellular transition. For example, targeting of gene MFAP5 was recently found to enhance chemosensitivity in ovarian and pancreatic cancers [40]. Specifically, the author states that "MFAP5 blockade suppresses fibrosis through downregulating of fibrosis-related genes such as COL11A1." Consistently, we found MFAP5 as one of the most highly associated genes with COL11A1 (Table 2).

As mentioned earlier, genes SFRP4 and RARRES1 are transiently expressed in Group 2 of Table 6 , suggesting that they can be investigated for inhibiting the cellular transition. Consistently, gene RARRES1 (aka TIG1) was found to play an important, though not yet clear, role in regulating proliferation and differentiation of adipose tissue derived mesenchymal stem cells [24], while SFRP4 is a Wnt pathway regulator whose expression has been found associated with various cancer types $[41,42]$.

Of particular interest as potential drivers are noncoding RNAs due to their typical regulatory role. Because the expression of these genes is not accurately captured by scRNA-seq technology, we did a thorough analysis of the full set of The Cancer Genome Atlas (TCGA) pan-cancer data. For the RNA sequencing and miRNA sequencing dataset of each cancer type, we removed the genes in which more than $50 \%$ of the samples have zero counts. Then we performed quantile normalization using the limma package [43] (v3.40.6) on $\log 2$ transformed counts. In each of the 
33 cancer types, we ranked all protein-coding genes in terms of the association (using the metric of mutual information) of their expression with that of gene COL11A1. We excluded the 11 cancer types ( $L G G, S K C M, S A R C, L A M L, P C P G, G B M, T G C T, T H Y M, A C C, U V M, U C S)$ in which neither THBS2 nor INHBA was among the 50 top-ranked genes, because of the absence of significant amounts of COL11A1-expressing CAFs in those samples (1st sheet in S9 Table). In each of the remaining 22 cancer types, we then ranked all long noncoding RNAs (lncRNAs) and microRNAs (miRNAs) in terms of their association with COL11A1 (2nd and 3rd sheets in S9 Table). Finally, we did pan-cancer sorting of all lncRNAs and miRNAs in terms of the median rank of all lncRNAs and miRNAs (4th sheet in S9 Table).

We found that LINC01614 represents a particularly promising therapeutic target. It had a perfect score of 1 in the pan-cancer sorting list, being strikingly top-ranked (top1) in 14 (BRCA, UCEC, KIRC, HNSC, LUAD, LUAD, LUSC, OV, STAD, ESCA, PAAD, MESO, DLBC, CHOL) out of the 22 cancer types (2nd sheet in S9 Table). In fact, the association of LINC01614 was even higher than that of marker protein-coding gene INHBA. We also found that the three top-ranked miRNAs were $m i R-199 a-1, m i R-199 b, m i R-199 a-2$. The associated $m i R-214$ is also very highly ranked (3rd sheet in S9 Table).

\section{Discussion}

One contribution of our work is that it provides an explanation to the fact that adipose tissue contributes to the development and progression of cancer as a result of its interaction with malignant cells [44-46].

Another contribution is that it sheds light on one important mechanism within the heterogeneous tumor microenvironment, which also contains fibroblasts that do not participate in the transition. 
There are several published results, often conflicting with each other, identifying various distinct subtypes of fibroblasts, resulting from computational clustering methods following dimensionality reduction, or using particular marker genes (such as $\alpha S M A$, which, interestingly we found that is not consistently expressed in the COL11A1-expressing CAFs, therefore it should not be used as its marker), which represent an approximation of biological reality. Examples include the hC1 and hC0 clusters in [47], the C9 and C10 clusters in [39], the CAF2 and CAF1 clusters in [33], the $\mathrm{iCAF}$ and myCAF clusters in $[48,49]$ and the $\mathrm{iCAF}$ an mCAF clusters in [50]. A review of such results in pancreatic cancer appears in [51].

As an example of conflicting results, the "iCAFs" identified in [50] are identical to the normal ASCs (fig. 3b of [50]) as evidenced by the list of its differentially expressed genes (PTGDS, LUM, $C F D, F B L N 1, A P O D, D C N, C X C L 14, S F R P 2, M M P 2$, all of which appear in Table 5, further validating the ASC signature. Therefore, this identified cluster contains mainly normal cells at the origin of the transition, which should not even be called CAFs. On the other hand, the iCAFs identified in [49] also have significant overlap in their marker composition with the ASCs (such as $C F D, D P T$ and $C X C L 12$ ), while omitting essential ASC markers such as APOD, and including additional genes.

On the other hand, our work is consistent with, and complementary to the results of [47] focusing on the immunotherapy response, in which the presence of the "TGF-beta CAFs" was inferred by an 11-gene signature consisting of MMP11, COL11A1, C1QTNF3, CTHRC1, COL12A1, COL10A1, COL5A2, THBS2, AEBP1, LRRC15, ITGA11. This population apparently represents the COL11A1-expressing CAF endpoint of the transition, and gene LRRC15 was selected as the representative gene based on the fact that it was found to be the most differentially expressed gene between CAFs and normal tissue fibroblasts in mouse models. Indeed, LRRC15 is a key member 
of the COL11A1-expressing CAF signature (table 4 of [1]) and we also found that COL11A1 is the highest associated gene to LRRC15 in the Group 3 PDAC patients,

In our work we used a detailed gene association-based scrutiny of all our results, including numerous color-coded scatter plots, observing and confirming the presence of cells expressing particular combinations of genes, rather than blindly accepting clustering results. We believe that this nontraditional computational methodology, when used on rich single-cell data, represents a paradigm shift in which systems biology alone can be trusted, by itself, for producing reliable results. We hope that our results will give rise to testable hypotheses that could eventually lead to the development of pan-cancer therapeutics inhibiting the ASC to COL11A1-expressing CAF transition.

\section{Materials and methods}

\subsection{Datasets availability}

The pancreatic dataset [21] was downloaded from the Genome Sequence Archive with accession number CRA001160. The four validation datasets of other cancer types are also publicly available: HNSCC [33] (GSE103322), ovarian [34] (GSE118828), lung cancer [35] (E-MTAB-6149 and EMTAB-6653), breast cancer [36] (GSE118389). We excluded samples from lymph nodes. The numbers of patients included in these datasets are 35 (PDAC), 18 (HNSCC), 9 (ovarian), 5 (lung), and 6 (breast).

\subsection{Data processing and cell identification}

We applied the Seurat (v3.1.4) [52] R package to process the gene expression matrix and characterize the cell type identity for each scRNA-seq dataset. The count matrix was normalized 
and $\log$ transformed by using the NormalizeData function. We selected the 2,000 most variable features and then performed principal component analysis (PCA) followed by applying an unsupervised graph-based clustering approach. We used default parameter settings in all the above steps except that the resolution parameter in the FindCluster function is set to 1.0 to increase the granularity of downstream clustering. To identify differentially expressed genes for each cluster, we used the FindMarkers function. To characterize the identity of mesenchymal cells in each dataset, we made use of the expression of known markers: $L U M, D C N, C O L 1 A 1$ for fibroblasts, and $R G S 5$ for pericytes.

For the smaller-size datasets (ovarian, breast), we performed clustering once on all cells for mesenchymal cell identification. For datasets of larger size (PDAC, HNSCC, lung), we applied 'two-step clustering' to ensure accuracy: The first step was initial clustering within individual samples. Then we excluded samples with very few $(<20)$ detected fibroblasts and pooled the mesenchymal cells of the remaining samples together for a second clustering, which resulted in the final set of mesenchymal cells for the dataset. For the PDAC dataset, we included an additional step to remove low-quality cells, by retaining cells for which at least one of the corresponding markers had expression levels $\geq 3$.

\subsection{Mutual information}

Mutual information (MI) is a general measure of the association between two random variables [53]. We used a spline based estimator [54] to estimate MI values and normalized so the maximum possible value is 1 . The details of the estimation method are described in the paper introducing the attractor algorithm [18]. We used the getMI or getAllMIWz function implemented in the cafr R package with parameter negateMI $=$ TRUE. 


\subsection{Attractor-based analysis}

For single dataset, we applied the attractor finding algorithm using the findAttractor function implemented in the cafr (v0.312) R package [18] with gene $L U M$ as seed. The exponent ( $a$ ) was set to different values for scRNA-seq datasets profiled from different protocols. For the analysis of UMI based (e.g. 10x) and full-length-based (e.g. Smart-seq2) datasets, we used $a=3$ and $a=5$, respectively. Briefly, the attractor finding algorithm iteratively finds mutually associated genes from an expression matrix, converging to the core of the co-expression mechanism. The association measure used is the normalized mutual information (as described above), which captures the general relationships (including nonlinear effects) between variables. To find the consensus attractor for multiple datasets, we applied the consensus version of the attractor finding algorithm as described in [55]. In the consensus version, the association measures between genes are evaluated as the weighted median of the corresponding measures taken from the individual datasets. The weights are proportional to the number of samples included in each individual dataset in log scale.

\subsection{Trajectory inference (TI) analysis}

We selected the Slingshot [32] method for TI analysis, based on its robustness and suggestions made by the benchmarking pipeline dynverse [56]. We used the raw counts as input and followed the Slingshot lineage analysis workflow (v1.4.0). To begin this process, Slingshot chose robustly expressed genes if it has at least 10 cells that have at least 1 read for each. After gene filtering, we proceeded to full quantile normalization. Following diffusion map dimensionality reduction, Gaussian mixture modelling was performed to classify cells. The final step of lineage inference analysis used the slingshot wrapper function. A cluster based minimum spanning tree was 
subjected to describe the lineage. After analyzing the global lineage structure, we fitted a generalized additive model (GAM) for pseudotime and computed $P$ values. Genes were ranked by $P$ values and variances. After running Slingshot, we identified genes whose expression values significantly vary over the derived pseudotime by using a GAM, allowing us to detect non-linear patterns in gene expression.

\subsection{Statistical analysis}

4.6.1 P value evaluation by permutation test. The significance of the consistency of $\mathrm{N}$ different attractors of size $\mathrm{M}$ was evaluated as follows. We randomly selected $\mathrm{M}$ genes out of the 24,005 genes to generate a random gene set, and we created $\mathrm{N}$ such random gene sets. Each time, we counted the number of genes common in all $\mathrm{N}$ gene sets, and we repeated this process ten million times. In these ten million experiments, it never occurred that there is one or more gene common in all $\mathrm{N}$ gene sets. Therefore, the corresponding $P$ value is less than $10^{-7}$.

4.6.2 Differential expression analysis. We used a Wilcoxon Rank Sum test by applying the FindMarkers function in Seurat to identify the differentially expressed (DE) genes between fibroblasts of different groups. DE genes with $\mid \log$ fold change $\mid>0.25$ and Bonferroni adjusted $P$ value $<0.1$ are considered as significant. The positive and negative DE genes are ranked separately in terms of the absolute values of their log fold-change.

\section{Acknowledgments}

The noncoding RNA-related results presented here are in whole based upon data generated by the TCGA Research Network: https://www.cancer.gov/tcga. 


\section{References}

1. Kim H, Watkinson J, Varadan V, Anastassiou D. Multi-cancer computational analysis reveals invasion-associated variant of desmoplastic reaction involving INHBA, THBS2 and COL11A1. BMC Med Genomics. 2010 Nov 3;3:51.

2. Schuetz CS, Bonin M, Clare SE, Nieselt K, Sotlar K, Walter M, et al. Progression-Specific Genes Identified by Expression Profiling of Matched Ductal Carcinomas In situ and Invasive Breast Tumors, Combining Laser Capture Microdissection and Oligonucleotide Microarray Analysis. Cancer Res. 2006 May 15;66(10):5278-86.

3. Bignotti E, Tassi RA, Calza S, Ravaggi A, Bandiera E, Rossi E, et al. Gene expression profile of ovarian serous papillary carcinomas: identification of metastasis-associated genes. American Journal of Obstetrics \& Gynecology. 2007 Mar 1;196(3):245.e1-245.e11.

4. Badea L, Herlea V, Dima SO, Dumitrascu T, Popescu I. Combined gene expression analysis of whole-tissue and microdissected pancreatic ductal adenocarcinoma identifies genes specifically overexpressed in tumor epithelia. Hepatogastroenterology. 2008 Dec;55(88):2016-27.

5. Wang M, Zhao Y, Zhang B. Efficient Test and Visualization of Multi-Set Intersections. Scientific Reports. 2015 Nov 25;5(1):16923.

6. Shen L, Yang M, Lin Q, Zhang Z, Zhu B, Miao C. COL11A1 is overexpressed in recurrent non-small cell lung cancer and promotes cell proliferation, migration, invasion and drug resistance. Oncol Rep. 2016 Aug;36(2):877-85. 
7. García-Pravia C, Galván JA, Gutiérrez-Corral N, Solar-García L, García-Pérez E, GarcíaOcaña M, et al. Overexpression of COL11A1 by Cancer-Associated Fibroblasts: Clinical Relevance of a Stromal Marker in Pancreatic Cancer. PLOS ONE. 2013 Oct 23;8(10):e78327.

8. Wu Y-H, Chang T-H, Huang Y-F, Chen C-C, Chou C-Y. COL11A1 confers chemoresistance on ovarian cancer cells through the activation of Akt/c/EBP $\beta$ pathway and PDK1 stabilization. Oncotarget. 2015 Sep 15;6(27):23748-63.

9. Chen P-C, Tang C-H, Lin L-W, Tsai C-H, Chu C-Y, Lin T-H, et al. Thrombospondin-2 promotes prostate cancer bone metastasis by the up-regulation of matrix metalloproteinase- 2 through down-regulating miR-376c expression. J Hematol Oncol [Internet]. 2017 Jan 25 ;10. Available from: https://www.ncbi.nlm.nih.gov/pmc/articles/PMC5264454/

10. Seder CW, Hartojo W, Lin L, Silvers AL, Wang Z, Thomas DG, et al. INHBA overexpression promotes cell proliferation and may be epigenetically regulated in esophageal adenocarcinoma. J Thorac Oncol. 2009 Apr;4(4):455-62.

11. Wang Q, Wen Y-G, Li D-P, Xia J, Zhou C-Z, Yan D-W, et al. Upregulated INHBA expression is associated with poor survival in gastric cancer. Med Oncol. 2012 Mar;29(1):7783.

12. Tu H, Li J, Lin L, Wang L. COL11A1 Was Involved in Cell Proliferation, Apoptosis and Migration in Non-Small Cell Lung Cancer Cells. J Invest Surg. 2020 Nov 5;1-6.

13. Wang X, Zhang L, Li H, Sun W, Zhang H, Lai M. THBS2 is a Potential Prognostic Biomarker in Colorectal Cancer. Scientific Reports. 2016 Sep 16;6(1):33366. 
14. Li X, Yu W, Liang C, Xu Y, Zhang M, Ding X, et al. INHBA is a prognostic predictor for patients with colon adenocarcinoma. BMC Cancer. 2020 Apr 15;20(1):305.

15. Seder CW, Hartojo W, Lin L, Silvers AL, Wang Z, Thomas DG, et al. Upregulated INHBA Expression May Promote Cell Proliferation and Is Associated with Poor Survival in Lung Adenocarcinoma. Neoplasia. 2009 Apr 1;11(4):388-96.

16. Verhaak RGW, Tamayo P, Yang J-Y, Hubbard D, Zhang H, Creighton CJ, et al. Prognostically relevant gene signatures of high-grade serous ovarian carcinoma. J Clin Invest. 2013 Jan 2;123(1):517-25.

17. Moffitt RA, Marayati R, Flate EL, Volmar KE, Loeza SGH, Hoadley KA, et al. Virtual microdissection identifies distinct tumor- and stroma-specific subtypes of pancreatic ductal adenocarcinoma. Nature Genetics. 2015 Oct;47(10):1168-78.

18. Cheng W-Y, Ou Yang T-H, Anastassiou D. Biomolecular events in cancer revealed by attractor metagenes. PLoS Comput Biol. 2013;9(2):e1002920.

19. Cheng W-Y, Ou Yang T-H, Anastassiou D. Development of a Prognostic Model for Breast Cancer Survival in an Open Challenge Environment. Science Translational Medicine. 2013 Apr 17;5(181):181ra50-181ra50.

20. McCarthy N. Rising to the challenge. Nature Reviews Cancer. 2013 Jun;13(6):378-378.

21. Peng J, Sun B-F, Chen C-Y, Zhou J-Y, Chen Y-S, Chen H, et al. Single-cell RNA-seq highlights intra-tumoral heterogeneity and malignant progression in pancreatic ductal adenocarcinoma. Cell Research. 2019 Sep;29(9):725-38. 
22. Boquest AC, Shahdadfar A, Frønsdal K, Sigurjonsson O, Tunheim SH, Collas P, et al. Isolation and Transcription Profiling of Purified Uncultured Human Stromal Stem Cells: Alteration of Gene Expression after In Vitro Cell Culture. Mol Biol Cell. 2005 Mar;16(3):1131-41.

23. Vijay J, Gauthier M-F, Biswell RL, Louiselle DA, Johnston JJ, Cheung WA, et al. Singlecell analysis of human adipose tissue identifies depot- and disease-specific cell types. Nature Metabolism. 2020 Jan;2(1):97-109.

24. Ohnishi S, Okabe K, Obata H, Otani K, Ishikane S, Ogino H, et al. Involvement of tazaroteneinduced gene 1 in proliferation and differentiation of human adipose tissue-derived mesenchymal stem cells. Cell Prolif. 2009 Feb 24;42(3):309-16.

25. Jing C, El-Ghany MA, Beesley C, Foster CS, Rudland PS, Smith P, et al. Tazarotene-Induced Gene 1 (TIG1) Expression in Prostate Carcinomas and Its Relationship to Tumorigenicity. J Natl Cancer Inst. 2002 Apr 3;94(7):482-90.

26. Oldridge EE, Walker HF, Stower MJ, Simms MS, Mann VM, Collins AT, et al. Retinoic acid represses invasion and stem cell phenotype by induction of the metastasis suppressors RARRES1 and LXN. Oncogenesis. 2013 Apr;2(4):e45-e45.

27. Kashiwagi M, Friess H, Uhl W, Berberat P, Abou-Shady M, Martignoni M, et al. Group II and IV phospholipase A2 are produced in human pancreatic cancer cells and influence prognosis. Gut. 1999 Oct;45(4):605-12. 
28. Buhmeida A, Bendardaf R, Hilska M, Laine J, Collan Y, Laato M, et al. PLA2 (group IIA phospholipase A2) as a prognostic determinant in stage II colorectal carcinoma. Annals of Oncology. 2009 Jul 1;20(7):1230-5.

29. Cai H, Chiorean EG, Chiorean MV, Rex DK, Robb BW, Hahn NM, et al. Elevated Phospholipase A2 Activities in Plasma Samples from Multiple Cancers. PLOS ONE. 2013 Feb 22;8(2):e57081.

30. Nallanthighal S, Rada M, Heiserman JP, Cha J, Sage J, Zhou B, et al. Inhibition of collagen XI alpha 1-induced fatty acid oxidation triggers apoptotic cell death in cisplatin-resistant ovarian cancer. Cell Death \& Disease. 2020 Apr 20;11(4):1-12.

31. Koundouros N, Poulogiannis G. Reprogramming of fatty acid metabolism in cancer. British Journal of Cancer. 2020 Jan;122(1):4-22.

32. Street K, Risso D, Fletcher RB, Das D, Ngai J, Yosef N, et al. Slingshot: cell lineage and pseudotime inference for single-cell transcriptomics. BMC Genomics. 2018 Jun $19 ; 19(1): 477$.

33. Puram SV, Tirosh I, Parikh AS, Patel AP, Yizhak K, Gillespie S, et al. Single-Cell Transcriptomic Analysis of Primary and Metastatic Tumor Ecosystems in Head and Neck Cancer. Cell. 2017 Dec 14;171(7):1611-1624.e24.

34. Shih AJ, Menzin A, Whyte J, Lovecchio J, Liew A, Khalili H, et al. Identification of grade and origin specific cell populations in serous epithelial ovarian cancer by single cell RNAseq. PLOS ONE. 2018 Nov 1;13(11):e0206785. 
35. Lambrechts D, Wauters E, Boeckx B, Aibar S, Nittner D, Burton O, et al. Phenotype molding of stromal cells in the lung tumor microenvironment. Nature Medicine. 2018 Aug;24(8):1277-89.

36. Karaayvaz M, Cristea S, Gillespie SM, Patel AP, Mylvaganam R, Luo CC, et al. Unravelling subclonal heterogeneity and aggressive disease states in TNBC through single-cell RNA-seq. Nature Communications. 2018 Sep 4;9(1):3588.

37. Vázquez-Villa F, García-Ocaña M, Galván JA, García-Martínez J, García-Pravia C, Menéndez-Rodríguez P, et al. COL11A1/(pro)collagen 11A1 expression is a remarkable biomarker of human invasive carcinoma-associated stromal cells and carcinoma progression. Tumor Biol. 2015 Apr 1;36(4):2213-22.

38. Jia D, Liu Z, Deng N, Tan TZ, Huang RY-J, Taylor-Harding B, et al. A COL11A1-correlated pan-cancer gene signature of activated fibroblasts for the prioritization of therapeutic targets. Cancer Lett. 2016 28;382(2):203-14.

39. Qian J, Olbrecht S, Boeckx B, Vos H, Laoui D, Etlioglu E, et al. A pan-cancer blueprint of the heterogeneous tumor microenvironment revealed by single-cell profiling. Cell Research. 2020 Jun 19;1-18.

40. Yeung T-L, Leung CS, Yip K-P, Sheng J, Vien L, Bover LC, et al. Anticancer Immunotherapy by MFAP5 Blockade Inhibits Fibrosis and Enhances Chemosensitivity in Ovarian and Pancreatic Cancer. Clin Cancer Res. 2019 Nov 1;25(21):6417-28. 
41. Drake J, Shearwood AM, White J, Friis R, Zeps N, Charles A, et al. Expression of secreted frizzled-related protein 4 (SFRP4) in primary serous ovarian tumours. Eur J Gynaecol Oncol. 2009;30(2):133-41.

42. Sandsmark E, Andersen MK, Bofin AM, Bertilsson H, Drabløs F, Bathen TF, et al. SFRP4 gene expression is increased in aggressive prostate cancer. Scientific Reports. 2017 Oct 27;7(1):14276.

43. Ritchie ME, Phipson B, Wu D, Hu Y, Law CW, Shi W, et al. limma powers differential expression analyses for RNA-sequencing and microarray studies. Nucleic Acids Res. 2015 Apr 20;43(7):e47.

44. Quail DF, Dannenberg AJ. The obese adipose tissue microenvironment in cancer development and progression. Nature Reviews Endocrinology. 2019 Mar;15(3):139-54.

45. Lengyel E, Makowski L, DiGiovanni J, Kolonin MG. Cancer as a Matter of Fat: The Crosstalk between Adipose Tissue and Tumors. Trends in Cancer. 2018 May 1;4(5):374-84.

46. Cozzo AJ, Fuller AM, Makowski L. Contribution of Adipose Tissue to Development of Cancer. Compr Physiol. 2017 Dec 12;8(1):237-82.

47. Dominguez CX, Müller S, Keerthivasan S, Koeppen H, Hung J, Gierke S, et al. Single-Cell RNA Sequencing Reveals Stromal Evolution into LRRC15+ Myofibroblasts as a Determinant of Patient Response to Cancer Immunotherapy. Cancer Discov. 2020 Feb $1 ; 10(2): 232-53$. 
48. Öhlund D, Handly-Santana A, Biffi G, Elyada E, Almeida AS, Ponz-Sarvise M, et al. Distinct populations of inflammatory fibroblasts and myofibroblasts in pancreatic cancer. J Exp Med. 2017 Mar 6;214(3):579-96.

49. Elyada E, Bolisetty M, Laise P, Flynn WF, Courtois ET, Burkhart RA, et al. Cross-Species Single-Cell Analysis of Pancreatic Ductal Adenocarcinoma Reveals Antigen-Presenting Cancer-Associated Fibroblasts. Cancer Discov. 2019 Aug 1;9(8):1102-23.

50. Chen Z, Zhou L, Liu L, Hou Y, Xiong M, Yang Y, et al. Single-cell RNA sequencing highlights the role of inflammatory cancer-associated fibroblasts in bladder urothelial carcinoma. Nature Communications. 2020 Oct 8;11(1):5077.

51. Helms E, Onate MK, Sherman MH. Fibroblast Heterogeneity in the Pancreatic Tumor Microenvironment. Cancer Discov. 2020 May 1;10(5):648-56.

52. Stuart T, Butler A, Hoffman P, Hafemeister C, Papalexi E, Mauck WM, et al. Comprehensive Integration of Single-Cell Data. Cell. 2019 Jun 13;177(7):1888-1902.e21.

53. Thomas M. Cover JAT. Elements Of Information Theory 2nd Ed [Internet]. 2006. Available from: http://archive.org/details/ElementsOfInformationTheory2ndEd

54. Daub CO, Steuer R, Selbig J, Kloska S. Estimating mutual information using B-spline functions--an improved similarity measure for analysing gene expression data. BMC Bioinformatics. 2004 Aug 31;5:118. 
55. Zhu K, Ou Yang T-H, Dorie V, Zheng T, Anastassiou D. Meta-analysis of expression and methylation signatures indicates a stress-related epigenetic mechanism in multiple neuropsychiatric disorders. Translational Psychiatry. 2019 Jan 22;9(1):1-12.

56. Saelens W, Cannoodt R, Todorov H, Saeys Y. A comparison of single-cell trajectory inference methods. Nature Biotechnology. 2019 May;37(5):547-54.

\section{Supplementary Material}

S1 Fig. Overview of the PDAC fibroblasts. A. 6,267 fibroblasts originated from 11 control pancreases and 23 tumor samples were petitioned into four groups X1-X4. Fractions of the fibroblasts were: 45\%, 38\%, $14 \%$, and 3\%. B. Table showing the top 20 DE genes for each cluster. C. Bar plots presenting the numbers of cells captured for each cluster.

S2 Fig. Trajectory analysis of 6,075 fibroblasts in PDAC dataset. A. Colors coded for pseudotime changing, red presenting the beginning of differentiation and blue presenting the end. B. Color-coded trajectory analysis of fibroblasts for annotated three clusters.

S3 Fig. Unsupervised clustering of four datasets from HNSCC, ovarian cancer, lung cancer and

breast cancer. A. t-SNE embedding of the whole HNSCC dataset. B. t-SNE embedding of the whole ovarian cancer dataset. C. t-SNE embedding of the mesenchymal cells from lung cancer dataset. D. t-SNE embedding of the mesenchymal cells from breast cancer dataset.

S1 Table. $L U M$-seeded attractors (top 100 genes) identified in each PDAC sample.

S2 Table. Differentially expressed genes of between the fibroblasts from different PDAC sample groups.

S3 Table. Top 100 genes of temporally expressed genes on the pseudotime variable.

S4 Table. Differentially expressed genes among different clusters of HNSCC dataset. 
S5 Table. $L U M$-seeded attractors (top 100 genes) from validating samples of other cancer types.

S6 Table. Differentially expressed genes among different clusters of ovarian cancer dataset.

S7 Table. Differentially expressed genes among different clusters of mesenchymal cells from lung cancer dataset.

S8 Table. Differentially expressed genes among different clusters of stromal cells from breast cancer dataset.

S9 Table. Ranked genes lists in terms of their association (mutual information) with gene COL11A by using the full set of pan-cancer TCGA bulk RNA-seq data. 